Archive for Organic Chemistry

\title{
Synthesis of dihydropyranones and dihydropyrano[2,3-d][1,3]dioxine-diones by cyclization reaction of Meldrum's acid with arylaldehydes and 1,3-dicarbonyls under thermal and ultrasound irradiation
}

\author{
Hossein Mehrabi*, Faezeh Najafian-Ashrafi, and Reza Ranjbar-Karimi \\ Department of Chemistry, Vali-e-Asr University of Rafsanjan, 77176, Rafsanjan, Iran \\ Email: mehraby h@yahoo.com
}

Received 11-24-2017

Accepted 01-28-2018

Published on line 03-15-2018

\section{Abstract}

The present paper deals with the synthesis of novel dihydropyranone and dihydropyrano[2,3-d][1,3]dioxinedione derivatives via one-pot three-component reaction between Meldrum's acid, arylaldehydes, and various 1,3-dicarbonyls in the presence of $\mathrm{KOH}$ as a base in $\mathrm{H}_{2} \mathrm{O}$ :EtOH under thermal and ultrasound irradiation. It was observed that ultrasound-assisted method gave $80-94 \%$ yields in 30-45 min as against 120-280 min required to get $60-82 \%$ yields by thermal method.

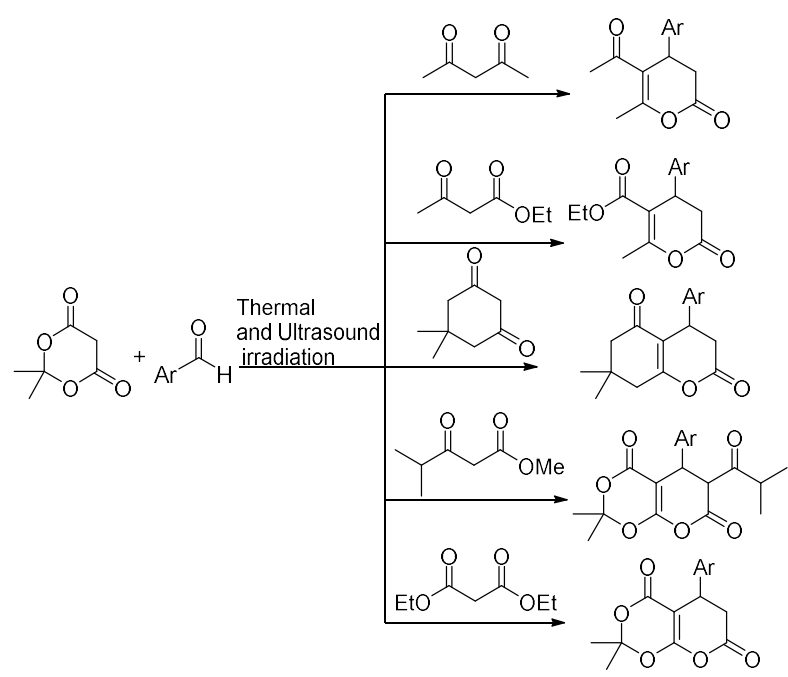

Keywords: One-pot three-component, dihydropyranones, dihydropyrano[2,3- $d][1,3]$ dioxine-dione, ultrasound irradiation 


\section{Introduction}

Pyran derivatives represent the key building blocks of many natural products, ${ }^{1-3}$ and constitute the core of valuable compounds exhibiting a broad spectrum of biological activities. ${ }^{4-8}$ Many of the $2 \mathrm{H}$-pyran-2-ones have been used as precursors for the synthesis of pharmacologically active compounds such as HIV protease inhibitors ${ }^{9}$ antifungals, ${ }^{10}$ cardiotonics, ${ }^{11}$ anticonvulsants, ${ }^{12}$ antimicrobials, ${ }^{13}$ pheromones, ${ }^{14}$ antitumor agents, ${ }^{15}$ and plant growth regulators. ${ }^{16}$ Also, $\alpha$-pyranones are important intermediates for the construction of pyridones, $\gamma$-lactones, benzenoid derivatives, and etc. ${ }^{17-23}$

Our literature survey revealed that there are some examples in the synthesis of 3,4-dihydro- $\alpha$-pyranones by several groups. ${ }^{24-31}$ But, little efforts have been paid to the synthesis of dihydropyranone and dihydropyrano[2,3- $d][1,3]$ dioxine-dione derivatives. ${ }^{32-38}$ To make the synthesis of this kind of compound in a more efficient and in continuation of our studies on one-pot multi-component reactions, ${ }^{39-41}$ we report here a one-pot three-component reaction of Meldrum's acid, arylaldehydes, and various 1,3-dicarbonyls in the presence of $\mathrm{KOH}$ as a base in $\mathrm{H}_{2} \mathrm{O}: \mathrm{EtOH}$ under thermal and ultrasound irradiation.

\section{Results and Discussion}

To find the optimal conditions, we studied the synthesis of 5-acetyl-6-methyl-4-phenyl-3,4-dihydro- $2 \mathrm{H}$-pyran2-one $\mathbf{4 a}$ from the condensation of Meldrum's acid 1, benzaldehyde 2a, and acetylacetone $\mathbf{3}$ under various reaction conditions (Table 1 ).

Table 1. Optimization of the reaction conditions

\begin{tabular}{llllll} 
& \multicolumn{1}{c}{} & & & & \\
\hline
\end{tabular}

aYields are given for isolated product.

The optimization of the reaction conditions, including the reaction solvent, bases, the reaction temperature, and the time of the reaction were investigated. First, various solvents were examined, the 
mixture of water and ethanol was proven to be preeminent solvent for this reaction. Then, we examined this reaction in the absence and presence of several bases (Table 1, entries 1-7). It was found that $\mathrm{KOH} \mathrm{(15 \% )}$ increased the yields from a trace to $60 \%$. Also, we examined the influence of different temperatures on this reaction. To our satisfaction, when the reaction was carried out at room temperature in 60 min, the product formed in $60 \%$ yield and at $50{ }^{\circ} \mathrm{C}$ in $30 \mathrm{~min}$, the product formed in $92 \%$ yield. But, under reflux condition in the same time the product was only formed in $65 \%$ yield (Table 1, entries 7,9 and 11). Finally, we observed that the amount of $\mathrm{KOH}$ more than and lower than $15 \%$ at $50{ }^{\circ} \mathrm{C}$ did not improve the yield (Table 1, entries 8-10). A series of experiments were performed to reveal that the optimal results were obtained when the reaction of 1 $(1.0 \mathrm{mmol})$ was conducted with benzaldehyde $2 \mathrm{a}(1.0 \mathrm{mmol})$, and acetylacetone $3(1.0 \mathrm{mmol})$ in $\mathrm{H}_{2} \mathrm{O}: \mathrm{EtOH}$ and $\mathrm{KOH}(15 \%)$ at $50{ }^{\circ} \mathrm{C}$ in $30 \mathrm{~min}$ (Table 1, entry 9). Under these optimized conditions the yield of 4 a reached $92 \%$. These optimized reaction conditions were then used to explore the scope of this novel transformation with various benzaldehydes, Meldrum's acid and acetylacetone to synthesize a series of 5-acetyl-6-methyl-4-aryl3,4-dihydro-2H-pyran-2-one derivatives $\mathbf{4 a - c}$ (Table 2) in good yields. Then the other 1,3-dicarbonyls such as 5, 7, 9 and 11 reacted with Meldrum's acid 1 and arylaldehydes 2 under the same conditions to give the corresponding dihydropyranones and dihydropyrano[2,3-d][1,3]dioxine-diones (Table 2).

Also, same reactions were performed under ultrasonic irradiation conditions. For example, the model reaction in $\mathrm{H}_{2} \mathrm{O}: \mathrm{EtOH}$ at $50{ }^{\circ} \mathrm{C}$ gave $4 \mathrm{a}$ in $72 \%$ yield after $120 \mathrm{~min}$, whereas the ultrasonic irradiation was rapid yielding $4 \mathrm{a}$ in $89 \%$ only within $30 \mathrm{~min}$. As shown in Table 2, the synthesis of dihydropyranones and dihydropyrano[2,3-d][1,3]dioxine-diones via the reaction of appropriate 1,3-dicarbonyls, Meldrum's acid with arylaldehydes were carried out in 80-94\% yield within 30-45 min under ultrasound irradiation, while without ultrasonic irradiation the products were obtained in $60-82 \%$ yield within $120-280$ min under thermal conditions. It is apparent that ultrasonic irradiation accelerates these transformations. Also, to investigate the effect of irradiation power on the yields and times of reactions, the model reaction was sonicated at 100, 200 and $300 \mathrm{~W}$. The results indicated that for the three power of ultrasound irradiation examined, there is no difference in the reaction yield and time. Therefore, $100 \mathrm{~W}$ of ultrasonic irradiation was sufficient to push the reaction forward.

The products $4 \mathbf{a}, \mathbf{4 b}$, and $\mathbf{6 a}$ are known and their structures were characterised by comparing their physical and spectral data with those of authentic samples. ${ }^{32-34}$ The other compounds are unknown to the best of our knowledge and were characterized by ${ }^{1} \mathrm{H} N M R,{ }^{13} \mathrm{C} \mathrm{NMR}, \mathrm{IR}$ and elemental analysis. For instance, the ${ }^{1} \mathrm{H}$ NMR spectrum of the compound $4 \mathrm{c}$ consisted of two singlet at $\delta=2.10$ and $2.34 \mathrm{ppm}$ for the two methyl protons. Two doublet at $\delta=2.67 \mathrm{ppm}$ with coupling constants of 2.2 and $15.8 \mathrm{~Hz}$ for the methylene protons was observed. In addition, two doublet at $\delta=3.15 \mathrm{ppm}$ with coupling constants of 7.0 and $15.8 \mathrm{~Hz}$ for another methylene protons was also observed. In addition, a doublet at $\delta=4.29 \mathrm{ppm}$ with a coupling constant of 6.4 $\mathrm{Hz}$ for the methine proton was also observed. A multiplet at $\delta=7.14-7.41 \mathrm{ppm}$ for the aromatic protons of the phenyl ring were also observed. The ${ }^{1} \mathrm{H}$ decoupled ${ }^{13} \mathrm{C}$ NMR spectrum of compound $4 \mathrm{c}$ showed 14 distinct signals in agreement with the proposed structure. Partial assignment of these resonances for the other products is given in the experimental section. 
Table 2. Synthesis of dihydropyranones and dihydropyrano[2,3-d][1,3]dioxine-diones under thermal $(A)$ and ultrasound irradiation (B)

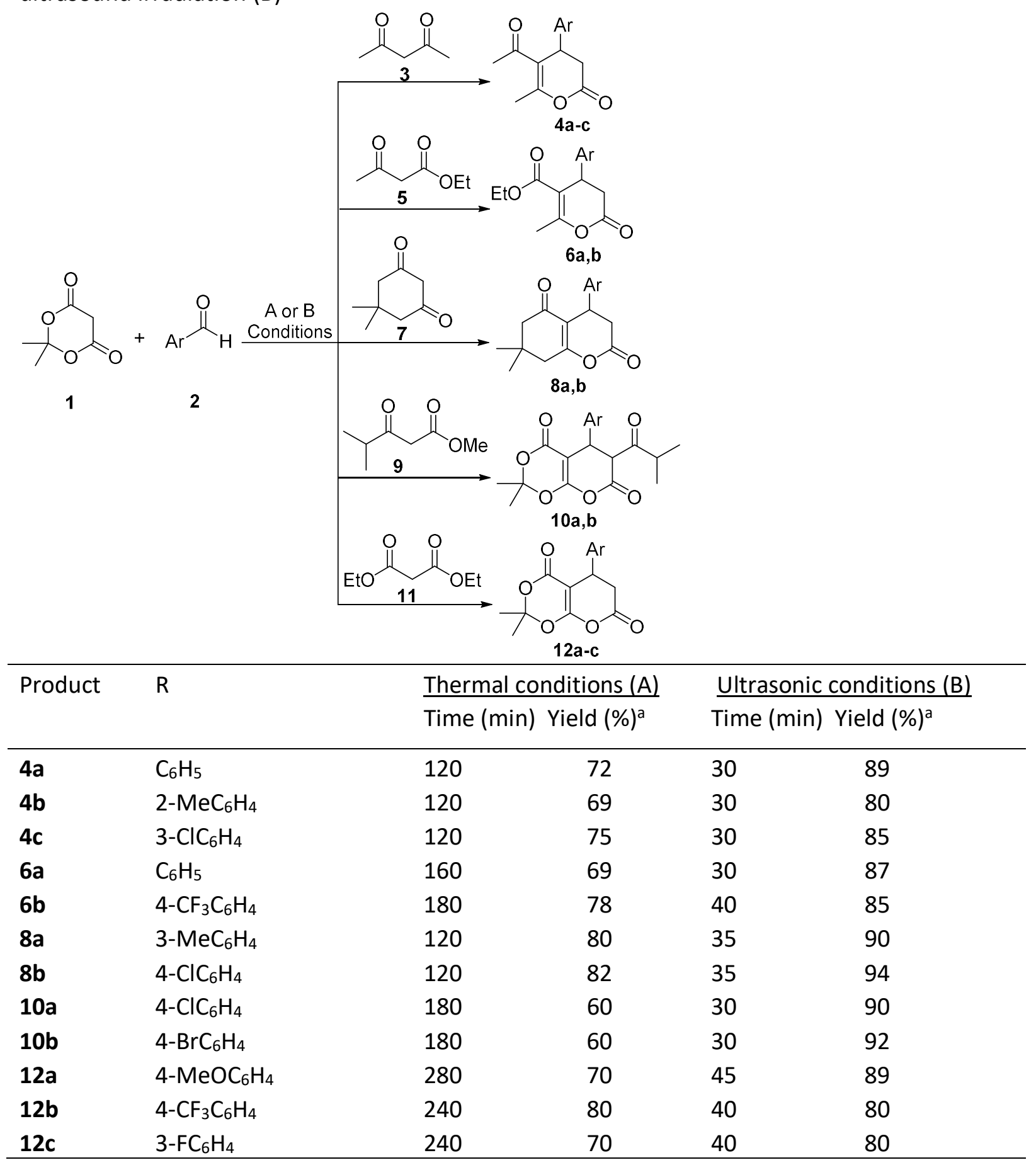

aYields are given for isolated product.

The possible mechanism for the synthesis of dihydropyranone and dihydropyrano[2,3- $d][1,3]$ dioxine-dione derivatives is illustrated in Scheme 1 . This conversion involves the initial reaction of Meldrum's acid $\mathbf{1}$ with arylaldehyde $\mathbf{2}$ to form the arylidene Meldrum's acid A. The arylidene Meldrum's acid A and 1,3-dicarbonyls such as 3, 5, 7, 9 and $\mathbf{1 1}$ produce the intermediates B, C, and D by a Michael nucleophilic addition. For 1,3dicarbonyls such as acetylacetone $\mathbf{3}$ and ethyl acetoacetate $\mathbf{5}$, the intermediate $\mathbf{B}$ then cyclises, eliminating acetone and carbon dioxide, in stages, affording 3,4-dihydropyranones 4 and $\mathbf{6}$. For the dimedone 7, the 
intermediate $\mathbf{C}$ then cyclises, eliminating acetone and carbon dioxide, in stages, affording 4,6,7,8tetrahydrochromene-2,5-dione 8. But, the methyl isobutyrylacetate $\mathbf{9}$ undergoes Michael type addition to arylidene Meldrum's acid $\mathbf{A}$ to give intermediate $\mathbf{D}$ which undergoes cyclization with loss of methanol to affording 5,6-dihydropyrano[2,3-d][1,3]dioxine-4,7-dione 10. Subsequently the diethyl malonate 11 undergoes Michael type addition to arylidene Meldrum's acid $\mathbf{A}$ to give intermediate $\mathbf{D}$ which undergoes cyclization with loss of ethanol and carbon dioxide to affording 5,6-dihydropyrano[2,3-d][1,3]dioxine-4,7-dione 12.

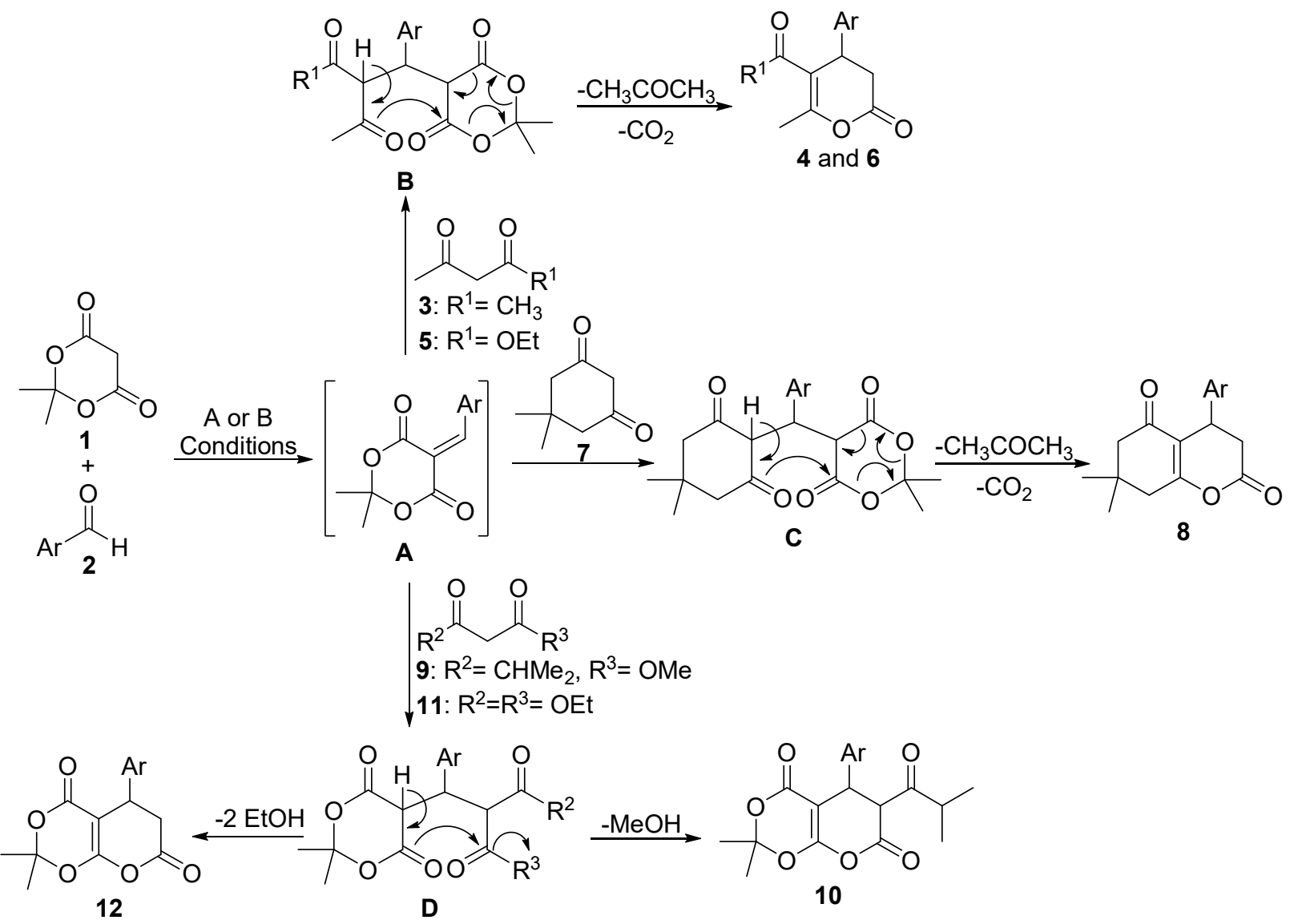

Scheme 1. Proposed mechanism for the synthesis of dihydropyranones and dihydropyrano[2,3-d][1,3]dioxinediones.

\section{Conclusions}

We have developed an efficient and facile method for the synthesis of dihydropyranone and dihydropyrano[2,3-d][1,3]dioxine-dione derivatives by the reaction of Meldrum's acid, arylaldehydes, and various 1,3-dicarbonyls under thermal and ultrasound irradiation conditions. The mild reaction conditions, low cost of the starting materials, operational simplicity and good yields are advantages of the protocol.

\section{Experimental Section}

General. All chemicals were purchased from Aldrich and Merck with high-grade quality, and used without any purification. All melting points were obtained by Bamslead Electrothermal 9200 apparatus and are uncorrected. The reactions were monitored by TLC and all yields refer to isolated products. ${ }^{1} \mathrm{H}$ and ${ }^{13} \mathrm{C}$ NMR 
spectra were recorded in $\mathrm{CDCl}_{3}$ on a Bruker $300 \mathrm{MHz}$ spectrometer. Infrared spectra were recorded on a Bruker FT-IR Equinax-55 spectrophotometer in $\mathrm{KBr}$ with absorption in $\mathrm{Cm}^{-1}$. Elemental analyses were performed using a Carlo Erba EA 1108 instrument. All products were characterized by their spectra and physical data.

General procedure for the synthesis of dihydropyranone and dihydropyrano[2,3-d][1,3]dioxine-dione derivatives

Thermal conditions. A mixture of the appropriate Meldrum's acid 1 (1.0 mmol), arylaldehydes $2(1.0 \mathrm{mmol})$, and various 1,3-dicarbonyls $(1.0 \mathrm{mmol})$ were stirred in $\mathrm{H}_{2} \mathrm{O}: \mathrm{EtOH}(4 \mathrm{~mL})$ in the presence of $\mathrm{KOH}(15 \%)$ as a base at $50{ }^{\circ} \mathrm{C}$ for an appropriate time. After completion of the reaction, determined by TLC, the solvent was removed under reduced pressure, and the resulting crude product was recrystallized from ethanol to give the pure compounds as a white solid.

Ultrasonic-irradiation conditions. A mixture of the appropriate Meldrum's acid $\mathbf{1}(1.0 \mathrm{mmol})$, arylaldehydes 2 (1.0 mmol), and various 1,3-dicarbonyls $(1.0 \mathrm{mmol})$ in $2 \mathrm{~mL}$ of $\mathrm{H}_{2} \mathrm{O}: \mathrm{EtOH}$ was irradiated under an ultrasonic processor at $25 \pm 1^{\circ} \mathrm{C}$ and $100 \mathrm{~W}$. After completion of the reaction, determined by TLC, the solvent was removed under reduced pressure, and the resulting crude product was recrystallized from ethanol to give the pure compounds as a white solid.

5-Acetyl-6-methyl-4-phenyl-3,4-dihydro-2H-pyran-2-one (4a). Mp 101-103 ${ }^{\circ} \mathrm{C}\left(102-104{ }^{\circ} \mathrm{C}, \mathrm{Lit}^{34}\right)$. IR v/cm ${ }^{-1}$ (KBr): 1724, 1690, 1442, 1358, 1270, 1226, 1186, 1147. ${ }^{1} \mathrm{H}$ NMR $\left(300 \mathrm{MHz}, \mathrm{CDCl}_{3}\right): \delta 2.10\left(\mathrm{~s}, 3 \mathrm{H}, \mathrm{COCH}_{3}\right), 2.34$ (s, 3H, CH$\left.)_{3}\right), 2.68\left(\mathrm{dd}, J 2.1\right.$ and $\left.15.7 \mathrm{~Hz}, 1 \mathrm{H}, \mathrm{CH}_{2}\right), 3.18\left(\mathrm{dd}, J 7.2\right.$ and $\left.15.7 \mathrm{~Hz}, 1 \mathrm{H}, \mathrm{CH}_{2}\right), 4.31(\mathrm{~d}, J 6.6 \mathrm{~Hz}, 1 \mathrm{H}, \mathrm{CH})$, 7.16-7.36 (m, 5H, ArH) ppm. ${ }^{13} \mathrm{C}$ NMR $\left(75 \mathrm{MHz} \mathrm{CDCl}_{3}\right): \delta$ 18.64, 29.70, 36.84, 37.33, 126.67, 127.35, 128.31, $129.07,140.61,159.62,166.30,197.62 \mathrm{ppm}$.

5-Acetyl-6-methyl-4-(o-tolyl)-3,4-dihydro-2H-pyran-2-one (4b). Mp 138-140 (137-139 ${ }^{\circ} \mathrm{C}$, Lit. $\left.{ }^{34}\right)$. IR v/cm ${ }^{-1}$ (KBr): 1772, 1693, 1622, 1425, 1380, 1353, 1343, 1247, 1192. ${ }^{1} \mathrm{H} \mathrm{NMR}\left(300 \mathrm{MHz}, \mathrm{CDCl}_{3}\right): \delta 2.10\left(\mathrm{~s}, 3 \mathrm{H}, \mathrm{COCH}_{3}\right)$, $2.27\left(\mathrm{~s}, 3 \mathrm{H}, \mathrm{CH}_{3}\right), 2.34\left(\mathrm{~s}, 3 \mathrm{H}, \mathrm{CH}_{3}\right), 2.67\left(\mathrm{dd}, J 2.2\right.$ and $\left.15.7 \mathrm{~Hz}, 1 \mathrm{H}, \mathrm{CH}_{2}\right), 3.16$ (dd, J 7.2 and $15.7 \mathrm{~Hz}, 1 \mathrm{H}, \mathrm{CH}_{2}$ ), 4.27 (d J $6.5 \mathrm{~Hz}, 1 \mathrm{H}, \mathrm{CH}), 6.93-7.24(\mathrm{~m}, 4 \mathrm{H}, \mathrm{ArH}) \mathrm{ppm} .{ }^{13} \mathrm{C} \mathrm{NMR}\left(75 \mathrm{MHz}, \mathrm{CDCl}_{3}\right): \delta$ 18.58, 21.00, 29.64, 36.83, $37.35,117.24,123.55,127.28,128.03,128.90,138.26,140.57,159.51,166.25,197.59$ ppm.

5-Acetyl-4-(3-chlorophenyl)-6-methyl-3,4-dihydro-2H-pyran-2-one (4c). Mp 188-190 ${ }^{\circ} \mathrm{C}$. IR v/cm-1 (KBr): 1762 , $1728,1594,1572,1477,1436,1392,1302,1284,1201 .{ }^{1} \mathrm{H}$ NMR $\left(300 \mathrm{MHz}, \mathrm{CDCl}_{3}\right): \delta 2.10\left(\mathrm{~s}, 3 \mathrm{H}, \mathrm{COCH}_{3}\right) 2.34$ (s, $3 \mathrm{H}, \mathrm{CH}_{3}$ ), 2.67 (dd, J 2.2 and $15.8 \mathrm{~Hz}, 1 \mathrm{H}, \mathrm{CH}_{2}$ ), 3.15 (dd, J 7.0 and $\left.15.8 \mathrm{~Hz}, 1 \mathrm{H}, \mathrm{CH}_{2}\right), 4.29(\mathrm{~d}, J 6.4 \mathrm{~Hz}, 1 \mathrm{H}, \mathrm{CH})$, 7.14-7.41 (m, 4H, ArH) ppm. $\left.{ }^{13} \mathrm{C} \mathrm{NMR} \mathrm{(75} \mathrm{MHz,} \mathrm{CDCl}\right): \delta=18.61,29.70,36.86,37.31,117.14,123.42,127.32$, $128.06,128.88,138.22,140.59,159.58,166.28,197.60$ ppm. Anal. Calcd for $\mathrm{C}_{14} \mathrm{H}_{13} \mathrm{ClO}_{3}$ (264.71): $\mathrm{C}, 63.53 ; \mathrm{H}$, 4.95. Found: C, 63.79; $\mathrm{H}, 4.97 \%$.

Ethyl 6-methyl-2-oxo-4-phenyl-3,4-dihydro-2H-pyran-5-carboxylate (6a). Mp 178-180 ${ }^{\circ} \mathrm{C}$ (Yellow oil, Lit. ${ }^{33}$ ). IR $\mathrm{v} / \mathrm{cm}^{-1}(\mathrm{KBr}): 1729,1610,1583,1458,1514,1377,1391,1289,1201,1183 .{ }^{1} \mathrm{H} \mathrm{NMR}\left(300 \mathrm{MHz}, \mathrm{CDCl}_{3}\right): \delta 1.08(\mathrm{t}$, J $\left.6.9 \mathrm{~Hz}, 3 \mathrm{H}, \mathrm{CH}_{3} \mathrm{CH}_{2}\right), 2.40\left(\mathrm{~s}, 3 \mathrm{H}, \mathrm{CH}_{3}\right), 2.68\left(\mathrm{dd}, J 2.1\right.$ and $\left.15.9 \mathrm{~Hz}, 1 \mathrm{H}, \mathrm{CH}_{2}\right), 3.20(\mathrm{dd}, J 7.5$ and $15.8 \mathrm{~Hz}, 1 \mathrm{H}$, $\left.\mathrm{CH}_{2}\right), 4.05\left(\mathrm{q}, J 6.9 \mathrm{~Hz}, 2 \mathrm{H}, \mathrm{OCH}_{2}\right), 4.22(\mathrm{~d}, J 6.9 \mathrm{~Hz}, 1 \mathrm{H}, \mathrm{CH}), 7.12-7.33(\mathrm{~m}, 5 \mathrm{H}, \mathrm{ArH}) \mathrm{ppm} .{ }^{13} \mathrm{C} \mathrm{NMR}(75 \mathrm{MHz}$, $\left.\mathrm{CDCl}_{3}\right): \delta 13.88,18.43,36.22,37.01,60.37,126.44,127.13,127.99,128.84,141.04,160.98,165.52,166.39$ ppm.

Ethyl 6-methyl-2-oxo-4-(4-(trifluoromethyl)phenyl)-3,4-dihydro-2H-pyran-5-carboxylate (6b) Mp 192-194 ${ }^{\circ} \mathrm{C}$. IR v/cm ${ }^{-1}(\mathrm{KBr}): 1733,1712,1619,1465,1376,1331,1246,1160,1118 .{ }^{1} \mathrm{H} \mathrm{NMR}\left(300 \mathrm{MHz}, \mathrm{CDCl}_{3}\right): \delta=1.10(\mathrm{t}, j$ $\left.=6.9 \mathrm{~Hz}, 3 \mathrm{H}, \mathrm{CH}_{3} \mathrm{CH}_{2}\right), 2.48\left(\mathrm{~s}, 3 \mathrm{H}, \mathrm{CH}_{3}\right), 2.70\left(\mathrm{dd}, J 2.1\right.$ and $\left.15.9 \mathrm{~Hz}, 1 \mathrm{H}, \mathrm{CH}_{2}\right), 3.23(\mathrm{dd}, J 7.5$ and $15.8 \mathrm{~Hz}, 1 \mathrm{H}$, $\mathrm{CH}_{2}$ ), $4.08\left(\mathrm{q}, J 6.9 \mathrm{~Hz}, 2 \mathrm{H}, \mathrm{OCH}_{2}\right), 4.25(\mathrm{~d}, J 6.9 \mathrm{~Hz}, 1 \mathrm{H}, \mathrm{CH}), 7.53(\mathrm{~d}, J 8.1 \mathrm{~Hz}, 2 \mathrm{H}, \mathrm{ArH}), 7.66(\mathrm{~d}, J 8.1 \mathrm{~Hz}, 2 \mathrm{H}$, ArH) ppm. ${ }^{13} \mathrm{C}$ NMR $\left(75 \mathrm{MHz}, \mathrm{CDCl}_{3}\right): \delta$ 13.91, 19.03, 36.16, 37.01, 61.35, 124.13, 125.94, 126.83, 128.09, $128.64,140.14,161.48,165.48,166.41$ ppm. Anal. Calcd for $\mathrm{C}_{16} \mathrm{H}_{15} \mathrm{~F}_{3} \mathrm{O}_{4}$ (328.29): $\mathrm{C}, 58.54 ; \mathrm{H}, 4.61$. Found: $\mathrm{C}$, 58.39; $\mathrm{H}, 4.58 \%$. 
7,7-Dimethyl-4-(m-tolyl)-4,6,7,8-tetrahydro-2H-chromene-2,5(3H)-dione (8a). Mp 118-120 ${ }^{\circ} \mathrm{C} . \quad \mathrm{IR} \quad \mathrm{v} / \mathrm{cm}^{-1}$ $(\mathrm{KBr}): 1786,1655,1373,1294,1158,1147,1112 .{ }^{1} \mathrm{H}$ NMR $\left(300 \mathrm{MHz}, \mathrm{CDCl}_{3}\right): \delta 1.06\left(\mathrm{~s}, 3 \mathrm{H}, \mathrm{CH}_{3}\right), 1.08(\mathrm{~s}, 3 \mathrm{H}$, $\left.\mathrm{CH}_{3}\right), 2.24(\mathrm{~d}, J 16.0 \mathrm{~Hz}, 1 \mathrm{H}, \mathrm{CHH}), 2.27\left(\mathrm{~s}, 3 \mathrm{H}, \mathrm{CH}_{3}\right), 2.33(\mathrm{~d}, J 16.0 \mathrm{~Hz}, 1 \mathrm{H}, \mathrm{CHH}), 2.51(\mathrm{~d}, J 17.8 \mathrm{~Hz}, 1 \mathrm{H}, \mathrm{CHH})$, $2.62\left(\mathrm{~d}, J 17.8 \mathrm{~Hz}, 1 \mathrm{H}, \mathrm{CHH}\right.$ ), 2.70 (dd, J 1.3 and $15.9 \mathrm{~Hz}, 1 \mathrm{H}, \mathrm{CH}_{2}$ ), 3.22 (dd, J 7.8 and $15.9 \mathrm{~Hz}, 1 \mathrm{H}, \mathrm{CH}_{2}$ ), 4.14 (d, J $7.5 \mathrm{~Hz}, 1 \mathrm{H}, \mathrm{CH}), 6.87-7.20(\mathrm{~m}, 4 \mathrm{H}, \mathrm{ArH}) \mathrm{ppm} .{ }^{13} \mathrm{C} \mathrm{NMR}\left(75 \mathrm{MHz}^{\mathrm{C} C D C l}\right): \delta 21.03,27.66,27.83,32.22,33.14$, $36.58,49.96,115.07,123.24,127.19,127.72,128.70,137.98,141.147,166.18,166.41,195.81$ ppm. Anal. Calcd for $\mathrm{C}_{18} \mathrm{H}_{20} \mathrm{O}_{3}$ (284.36): $\mathrm{C}, 76.03 ; \mathrm{H}, 7.09$. Found: $\mathrm{C}, 75.90 ; \mathrm{H}, 7.08 \%$.

4-(4-Chlorophenyl)-7,7-dimethyl-4,6,7,8-tetrahydro-2H-chromene-2,5(3H)-dione (8b). Mp $150-152{ }^{\circ} \mathrm{C}$. IR $\mathrm{v} / \mathrm{cm}^{-1}(\mathrm{KBr}): 1772,1655,1490,1373,1212,1112 .{ }^{1} \mathrm{H} \mathrm{NMR}\left(300 \mathrm{MHz}, \mathrm{CDCl}_{3}\right): \delta 1.04\left(\mathrm{~s}, 3 \mathrm{H}, \mathrm{CH}_{3}\right), 1.07(\mathrm{~s}, 3 \mathrm{H}$, $\left.\mathrm{CH}_{3}\right), 2.23(\mathrm{~d}, J 16.0 \mathrm{~Hz}, 1 \mathrm{H}, \mathrm{CHH}), 2.31(\mathrm{~d}, J 16.0 \mathrm{~Hz}, 1 \mathrm{H}, \mathrm{CHH}), 2.56(\mathrm{~d}, J 17.8 \mathrm{~Hz}, 1 \mathrm{H}, \mathrm{CHH}), 2.61(\mathrm{~d}, J 17.8 \mathrm{~Hz}$, $1 \mathrm{H}, \mathrm{CHH}), 2.71\left(\mathrm{dd}, J 1.3\right.$ and $\left.16.0 \mathrm{~Hz}, 1 \mathrm{H}, \mathrm{CH}_{2}\right), 3.26\left(\mathrm{dd}, J 7.9\right.$ and $\left.16.1 \mathrm{~Hz}, 1 \mathrm{H}, \mathrm{CH}_{2}\right), 4.18(\mathrm{~d}, J 7.5 \mathrm{~Hz}, 1 \mathrm{H}, \mathrm{CH})$, $7.13(\mathrm{~d}, J 7.2 \mathrm{~Hz}, 2 \mathrm{H}, \mathrm{ArH}), 7.36(\mathrm{~d}, J 7.2 \mathrm{~Hz}, 2 \mathrm{H}, \mathrm{ArH})$ ppm. ${ }^{13} \mathrm{C} \mathrm{NMR}\left(75 \mathrm{MHz}, \mathrm{CDCl}_{3}\right): \delta 27.71,27.79,32.19$, 32.60, 36.21, 49.87, 114.71, 128.36, 128.77, 131.68, 140.20, 166.24, 166.44, 195.83 ppm. Anal. Calcd for $\mathrm{C}_{17} \mathrm{H}_{17} \mathrm{ClO}_{3}$ (304.77): $\mathrm{C}, 67.00 ; \mathrm{H}, 5.62$. Found: $\mathrm{C}, 67.12 ; \mathrm{H}, 5.65 \%$.

5-(4-Chlorophenyl)-6-(1-hydroxy-2-methylprop-1-en-1-yl)-2,2-dimethyl-5,6-dihydro-4H,7H-pyrano[2,3-

d][1,3]dioxine-4,7-dione (10a). Mp 188-190 ${ }^{\circ} \mathrm{C} . \mathrm{IR} \mathrm{v} / \mathrm{cm}^{-1}$ (KBr): 1758, 1731, 1605, 1587, 1491, 1391, 1377, 1303, 1285, 1114. ${ }^{1} \mathrm{H}$ NMR $\left(300 \mathrm{MHz}, \mathrm{CDCl}_{3}\right): \delta 0.54\left(\mathrm{~s}, 6 \mathrm{H}, 2 \mathrm{CH}_{3}\right), 2.25\left(\mathrm{~s}, 6 \mathrm{H}, 2 \mathrm{CH}_{3}\right), 2.44(\mathrm{~m}, 1 \mathrm{H}, \mathrm{CH}), 3.44(\mathrm{~d}, J$ $15.2 \mathrm{~Hz}, 1 \mathrm{H}, \mathrm{CH}), 4.01(\mathrm{~d}, J 15.2 \mathrm{~Hz}, 1 \mathrm{H}, \mathrm{CH}), 6.92-7.27(\mathrm{~m}, 4 \mathrm{H}, \mathrm{ArH})$ ppm. ${ }^{13} \mathrm{C}$ NMR $\left(75 \mathrm{MHz}, \mathrm{CDCl}_{3}\right): \delta 18.56$, 20.95, 27.61, 42.32, 48.73, 59.78, 105.90, 125.30, 128.73, 128.89, 129.00, 137.36, 138.21, 164.78, 167.51, 206.54 ppm. Anal. Calcd for $\mathrm{C}_{19} \mathrm{H}_{19} \mathrm{ClO}_{6}$ (378.81): C, 60.24; H, 5.06. Found: C, 60.18; $\mathrm{H}, 5.03 \%$.

5-(4-Bromophenyl)-6-(1-hydroxy-2-methylprop-1-en-1-yl)-2,2-dimethyl-5,6-dihydro-4H,7H-pyrano[2,3-

d][1,3]dioxine-4,7-dione (10b). Mp 166-168 ${ }^{\circ} \mathrm{C} . \mathrm{IR} \mathrm{v} / \mathrm{cm}^{-1}$ (KBr): 1754, 1725, 1514, 1394, 1382, 1362, 1287, 1194. ${ }^{1} \mathrm{H}$ NMR $\left(300 \mathrm{MHz}, \mathrm{CDCl}_{3}\right): \delta 0.53\left(\mathrm{~s}, 6 \mathrm{H}, 2 \mathrm{CH}_{3}\right), 2.23\left(\mathrm{~s}, 6 \mathrm{H}, 2 \mathrm{CH}_{3}\right), 2.41(\mathrm{~m}, 1 \mathrm{H}, \mathrm{CH}), 3.46(\mathrm{~d}, J 14.7 \mathrm{~Hz}, 1 \mathrm{H}$, $\mathrm{CH}), 4.00$ (d, J $14.7 \mathrm{~Hz}, 1 \mathrm{H}, \mathrm{CH}), 7.02(\mathrm{~d}, J 7.8 \mathrm{~Hz}, 2 \mathrm{H}, \mathrm{ArH}), 7.16(\mathrm{~d}, J 7.8 \mathrm{~Hz}, 2 \mathrm{H}, \mathrm{ArH}) \mathrm{ppm} .{ }^{13} \mathrm{C} \mathrm{NMR}(75 \mathrm{MHz}$, $\left.\mathrm{CDCl}_{3}\right): \delta$ 19.04, 20.50, 27.72, 48.42, 59.98, 105.85, 128.04, 129.42, 134.27, 137.81, 138.19, 164.76, 167.61, 206.56 ppm. Anal. Calcd for $\mathrm{C}_{19} \mathrm{H}_{19} \mathrm{BrO}_{6}$ (423.26): C, 53.92; $\mathrm{H}, 4.52$. Found: $\mathrm{C}, 53.80 ; \mathrm{H}, 4.51 \%$.

5-(4-Methoxyphenyl)-2,2-dimethyl-5,6-dihydro-4H,7H-pyrano[2,3-d][1,3]dioxine-4,7-dione (12a). Mp 181$183^{\circ} \mathrm{C}$. IR v/cm ${ }^{-1}(\mathrm{KBr}): 1762,1728,1610,1514,1458,1391,1377,1315,1289,1252,1183 .{ }^{1} \mathrm{H} \mathrm{NMR}(300 \mathrm{MHz}$, $\mathrm{CDCl}_{3}$ ): $\delta 0.58\left(\mathrm{~s}, 6 \mathrm{H}, 2 \mathrm{CH}_{3}\right), 2.43\left(\mathrm{dd}, J 4.4\right.$ and $\left.31.5 \mathrm{~Hz}, 1 \mathrm{H}, \mathrm{CH}_{2}\right), 3.41\left(\mathrm{dd}, J 14.1\right.$ and $\left.31.3 \mathrm{~Hz}, 1 \mathrm{H}, \mathrm{CH}_{2}\right), 3.69(\mathrm{~s}$, $\left.3 \mathrm{H}, \mathrm{OCH}_{3}\right), 4.12(\mathrm{dd}, J 4.3$ and $13.9 \mathrm{~Hz}, 1 \mathrm{H}, \mathrm{CH}), 6.91(\mathrm{~d}, J 8.7 \mathrm{~Hz}, 2 \mathrm{H}, \mathrm{ArH}), 7.05(\mathrm{~d}, J 8.7 \mathrm{~Hz}, 2 \mathrm{H}, \mathrm{ArH}) \mathrm{ppm} .{ }^{13} \mathrm{C}$ $\operatorname{NMR}\left(75 \mathrm{MHz}_{\mathrm{CDCl}}\right.$ ): $\delta$ 27.87, 42.56, 47.99, 55.19, 60.35, 105.90, 114.33, 117.41, 129.32, 159.22, 164.89, 167.84, 206.71 ppm. Anal. Calcd for $\mathrm{C}_{16} \mathrm{H}_{16} \mathrm{O}_{6}$ (304.30): C, 63.15; $\mathrm{H}, 5.30$. Found: $\mathrm{C}, 63.27 ; \mathrm{H}, 5.33 \%$.

2,2-Dimethyl-5-(4-(trifluoromethyl)phenyl)-5,6-dihydro-4H,7H-pyrano[2,3-d][1,3]dioxine-4,7-dione (12b).

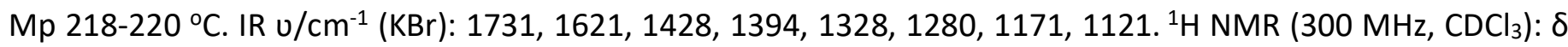
$0.50\left(\mathrm{~s}, 6 \mathrm{H}, 2 \mathrm{CH}_{3}\right), 2.42\left(\mathrm{dd}, J 4.2\right.$ and $31.0 \mathrm{~Hz}, 1 \mathrm{H}, \mathrm{CH}_{2}$ ), $3.42\left(\mathrm{dd}, J 15.1\right.$ and $\left.31.5 \mathrm{~Hz}, 1 \mathrm{H}, \mathrm{CH}_{2}\right), 4.00$ (dd, J 4.2 and $13.7 \mathrm{~Hz}, 1 \mathrm{H}, \mathrm{CH}), 7.39(\mathrm{~d}, J 8.1 \mathrm{~Hz}, 2 \mathrm{H}, \mathrm{ArH}), 7.78(\mathrm{~d}, J 8.1 \mathrm{~Hz}, 2 \mathrm{H}, \mathrm{ArH}) \mathrm{ppm} .{ }^{13} \mathrm{C} \mathrm{NMR}\left(75 \mathrm{MHz}, \mathrm{CDCl}_{3}\right): \delta$ 27.57, 41.71, 48.33, 106.18, 122.09, 126.06, 129.04, 129.40, 155.79, 164.41, 167.22, 205.51 ppm. Anal. Calcd for $\mathrm{C}_{16} \mathrm{H}_{13} \mathrm{~F}_{3} \mathrm{O}_{5}$ (342.27): $\mathrm{C}, 56.15 ; \mathrm{H}, 3.83$. Found: $\mathrm{C}, 56.21 ; \mathrm{H}, 3.79 \%$.

5-(3-Fluorophenyl)-2,2-dimethyl-5,6-dihydro-4H,7H-pyrano[2,3-d][1,3]dioxine-4,7-dione (12c). Mp 173-175 ${ }^{\circ} \mathrm{C}$. IR v/cm ${ }^{-1}(\mathrm{KBr}): 1733,1570,1475,1434,1395,1304,1200,1110 .{ }^{1} \mathrm{H} \mathrm{NMR}\left(300 \mathrm{MHz}, \mathrm{CDCl}_{3}\right): \delta 0.53(\mathrm{~s}, 6 \mathrm{H}$, $2 \mathrm{CH}_{3}$ ), 2.44 (dd, J 4.2 and $31.1 \mathrm{~Hz}, 1 \mathrm{H}, \mathrm{CH}_{2}$ ), 3.43 (dd, J 15.0 and $31.4 \mathrm{~Hz}, 1 \mathrm{H}, \mathrm{CH}_{2}$ ), 3.98 (dd, J 4.4 and $13.7 \mathrm{~Hz}$, $1 \mathrm{H}, \mathrm{CH}), 7.13-7.23(\mathrm{~m}, 4 \mathrm{H}, \mathrm{ArH}) \mathrm{ppm} .{ }^{13} \mathrm{C} \mathrm{NMR}\left(75 \mathrm{MHz}, \mathrm{CDCl}_{3}\right): \delta 27.75,41.94,48.22,106.17,115.32,124.60$, 131.31, 140.01, 160.57, 163.82, 164.51, 167.43, 205.79 ppm. Anal. Calcd for $\mathrm{C}_{15} \mathrm{H}_{13} \mathrm{FO}_{5}$ (292.26): C, 61.64; $\mathrm{H}$, 4.48. Found: C, $61.57 ; \mathrm{H}, 4.45 \%$. 


\section{References}

1. Ciblat, S.; Kim, J.; Stewart, C. A.; Wang, J.; Forgione, P.; Clyne, D.; Paquette, L. A. Org. Lett. 2007, 9, 719. https://doi.org/10.1021/ol063083i

2. Yang, R. Y.; Kizer, D.; Wu, H.; Volckova, E.; Miao, X. S.; Ali, S. M.; Tandon, M.; Savage, R. E.; Chan, T. C. K.; Ashwell, M. A. Bioorg. Med. Chem. 2008, 16, 5635.

https://doi.org/10.1016/i.bmc.2008.03.073

3. Tietze, L. F. Angew. Chem. Int. Ed. Engl. 1983, 22, 828. https://doi.org/10.1002/anie.198308281

4. Zhang, S.; Fernandez, F.; Hazeldine, S.; Deschamps, J.; Zhen, J.; Reith, M. E. A.; dutta, A. K. J. Med. Chem. 2006, 49, 4232.

5. Xu, Z. Q.; Pupek, K.; Suling, W. J.; Enache, L.; Flavin, M. T. Bioorg. Med. Chem. 2006, 14, 4610. https://doi.org/10.1016/i.bmc.2006.02.017

6. Souza, L. C.; Santos, A. F.; Goulart Sant Ana, A. E.; Oliveira Imbroisi, D. Bioorg. Med. Chem. 2004, $12,865$. https://doi.org/10.1016/i.bmc.2004.01.001

7. Ndi, C. P.; Semple, S. J.; Griesser, H. J.; Pyke, S. M.; Barton, M. D. Phytochemistry 2007, 68, 2684. https://doi.org/10.1016/i.phytochem.2007.05.039

8. Perez-Sacau, E.; Diaz-penate, R. G.; Estevez-Braun, A.; Ravelo, A. G.; Garcia-Castellano, J. M.; Pardo, L.; Campillo, M. J. Med. Chem. 2007, 50, 696.

https://doi.org/10.1021/jm060849b

9. Douglas, C. J.; Sklenicka, H. M.; Shen, H. C. Tetrahedron 1999, 55, 13683.

https://doi.org/10.1016/S0040-4020(99)00847-9

10. Claydon, N.; Allan, M.; Paquette, L. Trans. Br. Mycol. Soc. 1987, 88, 503. https://doi.org/10.1016/S0007-1536(87)80034-7

11. Liu, Z.; Meinwald. J. Org. Chem. 1996, 61, 6693. https://doi.org/10.1021/jo951394t

12. Aytemir, M. D.; Calis, U.; Ozalp, M. Arch. Pharm. Med. Chem. 2004, 337, 281. https://doi.org/10.1002/ardp.200200754

13. Yao, T.; Larock, R. C. J. Org. Chem. 2003, 68, 5936. https://doi.org/10.1021/jo034308v

14. Shi, X.; Leal, W. S.; Liu, Z.; Schrader, E.; Meinwald, Tetrahedron Lett. 1995, 36, 71. https://doi.org/10.1016/0040-4039(94)02214-V

15. Kondoh, M.; Usui, T.; Kobayashi, S. Cancer Lett. 1998, 126, 29. https://doi.org/10.1016/S0304-3835(97)00528-4

16. Tsuchiya, K.; Kobayashi, S.; Nishikiori, T.; Nakagawa, T.; Tatsuta, K. J Antibiot. 1997, 50, 259. https://doi.org/10.7164/antibiotics.50.259

17. Mandal, A. K.; Jawalkar, D. G. Tetrahedron Lett. 1986, 27, 99. https://doi.org/10.1016/S0040-4039(00)83951-9

18. Kume, T.; Iwasaki, H.; Yamamoto, Y.; Akiba, K. Tetrahedron Lett. 1988, 29, 3825 https://doi.org/10.1016/S0040-4039(00)82125-5

19. Mandal, A. K.; Jawalkar, D. J. J. Org. Chem. 1989, 54, 2364. https://doi.org/10.1021/jo00271a023

20. Robl, J. A. Tetrahedron Lett. 1990, 31, 3421. https://doi.org/10.1016/S0040-4039(00)97412-4 
21. Thang, S. H.; Rigg, D. J. Synth. Commun. 1993, 23, 2355. https://doi.org/10.1080/00397919308011120

22. Harrowven, D. C.; Hannam, J. C. Tetrahedron 1999, 55, 9333. https://doi.org/10.1016/S0040-4020(99)00495-0

23. Zhang, F. Y.; Corey, E. J. Org. Lett. 2000, 2, 1097. https://doi.org/10.1021/ol0056527

24. Itoh, K.; Hasegawa, M.; Tanaka, J.; Kanemasa, S. Org. Lett. 2005, 7, 979. https://doi.org/10.1021/ol047872g

25. De Sarkar, S.; Studer, A. Angew. Chem. Int. Ed. 2010, 49, 9266. https://doi.org/10.1002/anie.201004593

26. Fang, X. Q.; Chen, X. K.; Chi, Y. G. Org. Lett. 2011, 13, 4708. https://doi.org/10.1021/ol201917u

27. Rong, Z. Q.; Jia, M. Q.; You, S. L. Org. Lett. 2011, 13, 4080. https://doi.org/10.1021/ol201595f

28. Zhu, Z. Q.; Zheng, X. L.; Jiang, N. F.; Wan, X. L.; Xiao, J. C. Chem. Commun. 2011, 47, 8670. https://doi.org/10.1039/c1cc12778k

29. Mo, J. M.; Shen, L.; Chi, Y. G. Angew. Chem. Int. Ed. 2013, 52, 8588. https://doi.org/10.1002/anie.201302152

30. Rodriguez, H.; Coro, J.; Lam, A.; Salfran, E.; Rodriguez-Salarichs, J.; Suarez, M.; Albericio, F.; Martín, N. Arkivoc 2011, (ix), 125. http://dx.doi.org/10.3998/ark.5550190.0012.909

31. Suarez, M.; Verdecia, Y.; Ochoa, E.; Salfran, E.; Moran, L.; Martin, N.; Martinez, R.; Quinteiro, M.; Seoane,

C.; Soto, J. L.; Novoa, H.; Blaton, N.; Peeters, O. M.; Ranter, C. D. Eur. J. Org. Chem. 2000, 11, 2079. https://doi.org/10.1002/1099-0690(200006)2000:11<2079::AID-EJOC2079>3.0.CO;2-\#

32. Pratap, R.; Ram, V. J. Tetrahedron. 2017, 73, 2529. https://doi.org/10.1016/i.tet.2017.02.028

33. Albanese, D. C. M.; Gaggero, N. Eur J Org Chem. 2014, 40, 5631. https://doi.org/10.1002/ejoc.201402024

34. Wang, G.; Chen, X.; Miao, G. H.; Yao, W. J.; Ma, C. J. Org. Chem. 2013, 78, 6223. https://doi.org/10.1021/j0400950j

35. Xie, D.; Shen, D.; Chen, Q.; Zhou, J.; Zeng, X.; Zhong, G. J. Org. Chem. 2016, 81, 6136. https://doi.org/10.1021/acs.joc.6b01152

36. Zhao, B.; Du, D. Tetrahedron: Asymmetry. 2014, 25, 310. https://doi.org/10.1016/j.tetasy.2014.01.005

37. Abdolmohammadi, S.; Ghiasi, R.; Ahmadzadeh-Vatani, S. Z. Naturforsch B. 2016, 7, 777.

38. Tong-Shou, J.; Ai-Qing, W.; Zhao-Li, C.; Jian-She, Z.; Tong-Shuang, L. J. Chem. Res. 2004, 7, 457.

39. Mehrabi, H.; Najafian-Ashrafi, F.; Ranjbar-Karimi, R. J. Chem. Res. 2017, 41, 250. https://doi.org/10.3184/174751917X14902201357374

40. Mehrabi, H.; Mohebbi, A. Arkivoc 2016, (v), 89. http://dx.doi.org/10.3998/ark.5550190.p009.701

41. Mehrabi, H.; Anary-Abbasinejad, M.; Mirhashemi, F. Tetrahedron Lett. 2014, 55, 4310. https://doi.org/10.1016/i.tetlet.2014.06.025 